\title{
A MIXED-INTEGER LINEAR PROGRAMMING APPROACH FOR GLOBAL DISCRETE SIZE OPTIMIZATION OF FRAME STRUCTURES
}

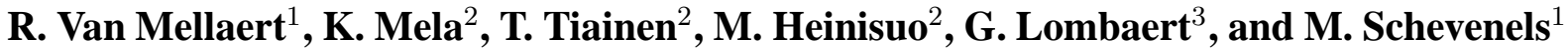 \\ ${ }^{1}$ Department of Architecture, Faculty of Engineering Science, KU Leuven, Belgium \\ Kasteelpark Arenberg 1 box 2431, 3001 Leuven \\ e-mail: roxane.vanmellaert@kuleuven.be \\ ${ }^{2}$ Department of Civil Engineering, Tampere University of Technology, Finland \\ ${ }^{3}$ Department of Civil Engineering, Faculty of Engineering Science, KU Leuven, Belgium
}

Keywords: Global optimization, discrete optimization, size optimization, frame structures, mixed-integer linear programs.

\begin{abstract}
This paper proposes a method to solve discrete size optimization problems of frame structures to global optimality. Global optimality is guaranteed by reformulating the optimization problem as a mixed-integer linear program (MILP) and solving it with the branch-andbound method. The presented mixed variable formulation extends the existing mixed variable formulation for size and topology optimization of truss structures. The MILP is obtained by adopting the simultaneous analysis and design approach. The variables consist of binary decision variables to select a profile section from the catalog, and state variables representing the member end forces. The equilibrium equations and member stiffness relations are included as constraints. The displacement and stress constraints are formulated such that for each member limit values are imposed at predefined locations along the member. The proposed method is applied to a three-bay three-story frame.
\end{abstract}




\section{Introduction}

In structural optimization, the problem formulation has a critical role as it imposes restrictions on the methods that can be used to solve the problem and it determines the applicability of the solution for the structural designer. If the principles of design, fabrication and economy [1] are followed, the problem formulation should contain as many requirements of these aspects as possible such that the need for post-processing the solution is minimized. On the other hand, incorporating all the requirements of the design codes, manufacturing practices and the economy of the structure into the problem as well-behaving (e.g. smooth) functions is very difficult in general, and enforcing them tend to make the problem intractable for most solution methods. Thus, it is fair to say that formulating an optimization problem is a compromise between meeting the needs of the structural designer and the capabilities of contemporary solution procedures.

For practical optimization of frame structures, the member profiles have to be chosen from a catalog of commercially available alternatives. When this feature is coupled with conventional formulations based on elastic structural analysis, the problem is not only nonlinear [2], but it also contains discrete design variables. The resulting mixed-integer nonlinear program (MINLP) can be treated by several optimization methods that have been proposed in the literature on discrete structural design of frames (for reviews, see $[3,4,5,6]$ ). However, these methods have in common that they cannot guarantee that the global optimum is found.

In a detailed review, Arora [6] discusses various methods for structural optimization with discrete variables. These include branch-and-bound for nonlinear problems, sequential linearization, dynamic rounding-off, penalty approach and various stochastic methods, among others. Some of the more recent approaches include the discrete Lagrangian-based algorithm [7], and a scheme based on the optimality criteria method [8].

Currently, evolutionary algorithms are widely used for solving discrete frame optimization problems. These methods include simulated annealing [9], genetic algorithms [10, 11], ant colony optimization [12], firefly algorithm [13], artificial bee colony algorithm [14], and particle swarm optimization [15]. The general idea is to explore the design space in a random fashion, thereby using information collected from previous analyses to gradually move towards a better performing design. Evolutionary algorithms owe their popularity to the fact that they are easy to understand and to implement. They can cope with discrete parameters and are able to take into account complex constraints. However, evolutionary algorithms converge slowly, involve algorithmic parameters that require careful tuning, and global optimality cannot be guaranteed since no conclusive convergence checks can be made.

This paper presents a method for global discrete size optimization of frame structures. The adopted approach is to reformulate the optimization problem into a mixed-integer linear program (MILP). In the classical approach for structural optimization the nested analysis and design (NAND) approach is employed [16]: in every iteration a finite element analysis is performed in order to obtain the state variables (the structural nodal displacements and the member end forces). In order to facilitate the reformulation of the optimization problem as an MILP, the simultaneous analysis and design (SAND) approach is adopted [16] in this study: the state variables are considered as additional design variables and the state equations (the equilibrium equations and member stiffness relations) are enforced by means of additional constraints. In addition, a set of binary decision variables is introduced for each member of the structure to select a profile from the profile section catalog. The obtained MILP can be solved to global optimality with well-established algorithms such as branch-and-bound methods. 
The MILP formulation approach has originally been proposed by Grossmann et al. [17] for discrete size optimization problems and is extended by Rasmussen et al. [18] for truss topology design problems. Mela [19] included member strength and buckling constraints specified by the Eurocode in the truss topology design problem. Van Mellaert et al. [20] included both the member and the joint constraints for size optimization of statically determinate trusses.

The formulation approach proposed in this paper differs from the formulation for truss optimization problems. In the formulation for truss structures, the variables consist of the binary decision variables, the displacement, and the normal forces. The internal resistance constraints are imposed by limiting the normal force of the member and displacement constraints are imposed by limiting the nodal displacements. For frame structures, the normal forces are replaced with the internal member end forces, including bending moments and shear forces. The proposed approach to take into account the member resistance and displacement constraints makes it possible to limit the relevant displacement components and stresses at several predefined locations of the members. These displacement and stress constraints are evaluated in a number of points using shape functions that interpolate the nodal displacements in order to calculate the stresses and displacements at the predefined locations of the members.

The paper is organized as follows. In section 2, the mixed-integer linear program of frame optimization problems is introduced: the design variables as well as the constraints are described. In section 3, the method is applied to a three-bay three-story frame.

\section{Mixed variable formulation}

In this section, the mixed integer linear program reformulation for frame structures is described. The design variables as well as the constraints of the optimization problem are introduced.

The proposed formulation is written for plane frames with prismatic members analysed by linear elastic theory. For simplicity, it is assumed that all members are made of the same material. Moreover, only a single loading condition is considered, but the formulation is easily extended to multiple loading conditions. The joints are assumed to be rigid, although hinged connections can be incorporated in the formulation as well.

Consider a frame structure defined by $n_{m}$ members and $n_{n}$ nodes with $n_{\text {dof }}$ degrees of freedom. The number of profile alternatives is $n_{s}$. Denote by $\mathcal{M}=\left\{1,2, \ldots, n_{m}\right\}$ and $\mathcal{C}=\left\{1,2, \ldots, n_{s}\right\}$ the index sets for members and profiles. Each member may have its own set of profile alternatives. The index set of profiles of member $i$ is denoted by $\mathcal{C}_{i} \subseteq \mathcal{C}$.

\subsection{Design variables}

The design variables include a vector with binary decision variables $\mathbf{y}$, a vector with continuous nodal displacement variables $\mathbf{u}$, and a vector with continuous force variables $\mathbf{q}$. The binary variables select a profile from the set of available alternatives. For each member $i$, profile $j$ is selected when the corresponding variable $y_{i j}=1$. Profile $j$ is not selected for member $i$ when the corresponding variable $y_{i j}=0$. The force variables represent the member end forces. This means that for each member $i$ and for each section $j$ the force variables are represented by the normal end forces $N_{1, i j}$ and $N_{2, i j}$, the shear end forces $V_{1, i j}$ and $V_{2, i j}$, and the bending moment end forces $M_{1, i j}$ and $M_{2, i j}$ as shown in figure 1. The member end forces for each member $i$ and for each section $j$ are collected in the vector $\mathbf{q}_{i j}$ :

$$
\mathbf{q}_{i j}=\left[\begin{array}{llllll}
N_{1, i j} & V_{1, i j} & M_{1, i j} & N_{2, i j} & V_{2, i j} & M_{2, i j}
\end{array}\right]^{\mathrm{T}}
$$




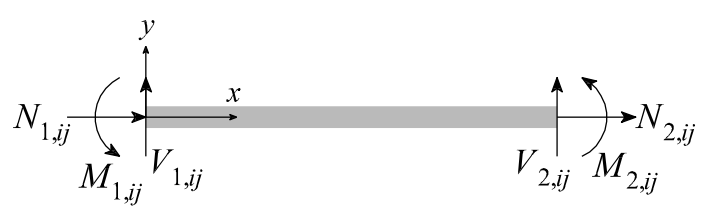

Figure 1: Member end forces $\mathbf{q}_{i j}$.

The vector with the design variables $\mathbf{x}$ is given by:

$$
\mathbf{x}=\left[\begin{array}{lll}
\mathbf{y}^{\mathrm{T}} & \mathbf{u}^{\mathrm{T}} & \mathbf{q}^{\mathrm{T}}
\end{array}\right]^{\mathrm{T}}, \quad \mathbf{y} \in \mathbb{B}^{n_{\mathrm{b}}}, \mathbf{u} \in \mathbb{R}^{n_{\mathrm{dof}}}, \mathbf{q} \in \mathbb{R}^{6 n_{\mathrm{b}}}
$$

The total number of binary decision variables is denoted by $n_{\mathrm{b}}=\sum_{i=1}^{n_{\mathrm{m}}} n_{\mathrm{s} i}$, where $n_{\mathrm{m}}$ is the total number of members in the structure, and $n_{\mathrm{s} i}$ is the total number of available sections for member $i$. The total number of degrees of freedom is denoted by $n_{\text {dof }}$. The total number of force variables is $6 n_{\mathrm{b}}$. The total number of design variables is calculated as $n_{\mathrm{dv}}=n_{\mathrm{dof}}+7 n_{\mathrm{b}}$.

\subsection{MILP}

The mixed-integer linear program for frame structures is given by equations (3) to (9):

$$
\min _{\mathbf{x}} \sum_{i \in \mathcal{M}} \sum_{j \in \mathcal{C}_{i}} c_{i j} y_{i j}
$$

such that

$$
\begin{array}{cl}
\sum_{j \in \mathcal{C}_{i}} y_{i j}=1 & \forall i \in \mathcal{M} \\
\sum_{i \in \mathcal{M}} \sum_{j \in \mathcal{C}_{i}} \mathbf{L}_{i}^{\mathrm{T}} \mathbf{T}_{i}^{\mathrm{T}} \mathbf{q}_{i j}=\mathbf{f} & \\
\left(1-y_{i j}\right) \underline{\mathbf{q}}_{i j}^{\prime} \leqslant \mathbf{K}_{i j} \mathbf{T}_{i} \mathbf{L}_{i} \mathbf{u}-\mathbf{q}_{i j} \leqslant\left(1-y_{i j}\right) \overline{\mathbf{q}}_{i j}^{\prime} & \forall i \in \mathcal{M}, \forall j \in \mathcal{C}_{i} \\
\underline{\mathbf{q}}_{i j}^{\prime} y_{i j} \leqslant \mathbf{q}_{i j} \leqslant \overline{\mathbf{q}}_{i j}^{\prime} y_{i j} & \forall i \in \mathcal{M}, \forall j \in \mathcal{C}_{i} \\
\underline{\mathbf{d}}_{i j}^{\prime}+\left(\underline{\mathbf{d}}_{i j}-\underline{\mathbf{d}}_{i j}^{\prime}\right) y_{i j} \leqslant \mathbf{N}_{i} \mathbf{T}_{i} \mathbf{L}_{i} \mathbf{u}+\tilde{\mathbf{d}}_{i j} \leqslant \overline{\mathbf{d}}_{i j}^{\prime}+\left(\overline{\mathbf{d}}_{i j}-\overline{\mathbf{d}}_{i j}^{\prime}\right) y_{i j} & \forall i \in \mathcal{M}, \forall j \in \mathcal{C}_{i} \\
\underline{\mathbf{s}}_{i j}^{\prime}+\left(\underline{\mathbf{s}}_{i j}-\underline{\mathbf{s}}_{i j}^{\prime}\right) y_{i j} \leqslant \mathbf{B}_{i j} \mathbf{T}_{i} \mathbf{L}_{i} \mathbf{u}+\tilde{\mathbf{s}}_{i j} \leqslant \overline{\mathbf{s}}_{i j}^{\prime}+\left(\overline{\mathbf{s}}_{i j}-\overline{\mathbf{s}}_{i j}^{\prime}\right) y_{i j} & \forall i \in \mathcal{M}, \forall j \in \mathcal{C}_{i}
\end{array}
$$

The objective function is given by equation (3). The subsequent equations are the constraints of the optimization problem. Equation (4) represents the profile selection. The equilibrium equations and the member stiffness relations are given by equation (5) and (6) respectively. Equation (7) ensures that the member forces are zero when profile $j$ is not selected for member $i$. The displacements at predefined locations of member $i$ are limited by the constraints given by equation (8). The stresses at predefined locations of member $i$ are limited by the constraints given by equation (9). In the following subsections the details of deriving these equations are described.

It is possible to account for multiple load cases by extending the MILP with additional nodal displacement and force variables for each load case and additional constraints: the constraints given by equation (5) to (9) are repeated for each load case considering the appropriate nodal displacement and forces variables [19]. In this paper, only one load case is assumed. 


\subsection{Objective function}

The objective function is given by equation (3):

$$
\min _{\mathbf{x}} \sum_{i \in \mathcal{M}} \sum_{j \in \mathcal{C}_{i}} c_{i j} y_{i j}
$$

where $c_{i j}$ is the cost of profile $j$ for member $i$. When the total weight of the structure is minimized, the cost of profile $j$ for member $i$ is defined as $c_{i j}=\rho L_{i} A_{i j}$, where $\rho$ is the density of the material, $L_{i}$ is the length of member $i$, and $A_{i j}$ is the section area of section $j$ for member $i$. In this paper a single material is used, but multiple materials can be adopted with the formulation.

\subsection{Selection constraints}

The constraint given by equation (4) ensures that a single section $j$ is chosen from the catalog $\mathcal{C}_{i}$ for member $i$.

$$
\sum_{j \in \mathcal{C}_{i}} y_{i j}=1 \quad \forall i \in \mathcal{M}
$$

\subsection{Equilibrium equations}

The nodal equilibrium is imposed by the equality constraints given by equation (5):

$$
\sum_{i \in \mathcal{M}} \sum_{j \in \mathcal{C}_{i}} \mathbf{L}_{i}^{\mathrm{T}} \mathbf{T}_{i}^{\mathrm{T}} \mathbf{q}_{i j}=\mathbf{f}
$$

where $\mathbf{L}_{i}$ is a $6 \times n_{\text {dof }}$ binary location matrix that maps the element degrees of freedom to the system degrees of freedom, $\mathbf{T}_{i}$ is a $6 \times 6$ transformation matrix that accounts for the orientation of the element [21], and $\mathbf{f}$ is the $n_{\text {dof }} \times 1$ nodal load vector. Element loads are taken into account as equivalent nodal loads in the nodal load vector $\mathbf{f}$.

\subsection{Member stiffness relations}

In addition to nodal equilibrium, the material law and compatibility conditions are needed in structural analysis. For trusses, Hooke's law and compatibility conditions can be written as a single equation, because the normal force is the only stress resultant appearing in the members [18]. As frame members have three (6 in 3D) stress resultants in each node, altogether six (12 in 3D) force-displacement relations are needed. Thus, the relation between the member end forces and the nodal displacements can be written as:

$$
\mathbf{q}_{i j}=\mathbf{K}_{i j} \mathbf{T}_{i} \mathbf{L}_{i} \mathbf{u} y_{i j} \quad \forall i \in \mathcal{M}, \forall j \in \mathcal{C}_{i}
$$

where $\mathbf{K}_{i j}$ is the element stiffness matrix:

$$
\mathbf{K}_{i j}=\left[\begin{array}{cccccc}
\frac{E A_{i j}}{L_{i}} & 0 & 0 & -\frac{E A_{i j}}{L_{i}} & 0 & 0 \\
0 & \frac{12 E I_{i j}}{L_{i}^{3}} & \frac{6 E I_{i j}}{L_{i}^{2}} & 0 & -\frac{12 E I_{i j}}{L_{i}^{3}} & \frac{6 E I_{i j}}{L_{i}^{2}} \\
0 & \frac{6 E I_{i j}}{L_{i}^{2}} & \frac{4 E I_{i j}}{L_{i}} & 0 & -\frac{6 E I_{i j}}{L_{i}^{2}} & \frac{2 E I_{i j}}{L_{i}} \\
-\frac{E A_{i j}}{L_{i}} & 0 & 0 & \frac{E A_{i j}}{L_{i}} & 0 & 0 \\
0 & -\frac{12 E I_{i j}}{L_{i}^{3}} & -\frac{6 E I_{i j}}{L_{i}^{2}} & 0 & \frac{12 E I_{i j}}{L_{i}^{3}} & -\frac{6 E I_{i j}}{L_{i}^{2}} \\
0 & \frac{6 E I_{i j}^{2}}{L_{i}^{2}} & \frac{2 E I_{i j}}{L_{i}} & 0 & -\frac{6 E I_{i j}}{L_{i}^{2}} & \frac{4 E I_{i j}}{L_{i}}
\end{array}\right]
$$


where $E$ is the Young's modulus of the material, $L_{i}$ is the length of member $i$, and $A_{i j}$ and $I_{i j}$ are the section area and second moment of area of profile $j$ for member $i$, respectively.

Equation (10) ensures that the force variables $\mathbf{q}_{i j}$ become zero when profile $j$ is not selected for member $i\left(y_{i j}=0\right)$ and $\mathbf{q}_{i j}=\mathbf{K}_{i j} \mathbf{T}_{i} \mathbf{L}_{i} \mathbf{u}$ when profile $j$ is selected for member $i\left(y_{i j}=1\right)$.

In a regular finite element analysis, the global stiffness matrix $\mathbf{K}$ is assembled by replacing $\mathbf{q}_{i j}$ in equation (5) with the expression given by equation (10). The resulting equilibrium equation can not be reformulated as a linear system of equations in terms of the design variables since the global stiffness matrix depends on the binary decision variables. Therefore, the linear nodal equilibrium equation (5) and the member stiffness relation equation (10) are adopted as separate constraints.

The member stiffness relation in equation (10) is nonlinear in terms of the design variables but can be equivalently reformulated as a set of linear inequality constraints by introducing artificial upper and lower bounds (big-M) [18] as equation (6):

$$
\left(1-y_{i j}\right) \underline{\mathbf{q}}_{i j}^{\prime} \leqslant \mathbf{K}_{i j} \mathbf{T}_{i} \mathbf{L}_{i} \mathbf{u}-\mathbf{q}_{i j} \leqslant\left(1-y_{i j}\right) \overline{\mathbf{q}}_{i j}^{\prime} \quad \forall i \in \mathcal{M}, \forall j \in \mathcal{C}_{i}
$$

In this equation, the force variables become equal to $\mathbf{q}_{i j}=\mathbf{K}_{i j} \mathbf{T}_{i} \mathbf{L}_{i} \mathbf{u}$ when profile $j$ is selected for member $i\left(y_{i j}=1\right)$. When profile $j$ is not selected for member $i\left(y_{i j}=0\right)$, the force variables do not become zero but are bounded by $\mathbf{K}_{i j} \mathbf{T}_{i} \mathbf{L}_{i} \mathbf{u}-\overline{\mathbf{q}}_{i j}^{\prime} \leqslant \mathbf{q}_{i j} \leqslant \mathbf{K}_{i j} \mathbf{T}_{i} \mathbf{L}_{i} \mathbf{u}-\underline{\mathbf{q}}_{i j}^{\prime}$. In order to ensure that the force variables become zero when profile $j$ is not selected for member $i$, an additional constraint given by equation (7) is introduced:

$$
\underline{\mathbf{q}}_{i j}^{\prime} y_{i j} \leqslant \mathbf{q}_{i j} \leqslant \overline{\mathbf{q}}_{i j}^{\prime} y_{i j} \quad \forall i \in \mathcal{M}, \forall j \in \mathcal{C}_{i}
$$

The artificial upper and lower bounds $\overline{\mathbf{q}}_{i j}^{\prime}$ and $\mathbf{q}_{i j}^{\prime}$ ensure feasibility when profile $j$ is not selected for member $i$ and are calculated as follows [22]:

$$
\begin{array}{rr}
\underline{\mathbf{q}}_{i j}^{\prime}=\min _{\mathbf{u}} \quad \mathbf{K}_{i j} \mathbf{T}_{i} \mathbf{L}_{i} \mathbf{u} \\
\text { s.t. } \quad \underline{\mathbf{u}} \leqslant \mathbf{u} \leqslant \overline{\mathbf{u}} \\
\overline{\mathbf{q}}_{i j}^{\prime}=\max _{\mathbf{u}} \quad \mathbf{K}_{i j} \mathbf{T}_{i} \mathbf{L}_{i} \mathbf{u} \\
\text { s.t. } \quad \underline{\mathbf{u}} \leqslant \mathbf{u} \leqslant \overline{\mathbf{u}}
\end{array}
$$

where $\underline{\mathbf{u}}$ and $\overline{\mathbf{u}}$ are the prescribed minimum and maximum allowed nodal displacements, respectively. Note that equations (12) and (13) are linear optimization problems with bound constraints, that can be solved without effort [22].

\subsection{Displacements along elements}

For truss structures, the loads are applied to the nodes and the normal forces remain constant along the members. For each member, the internal resistance constraints can therefore be imposed by limiting the normal force of the member and displacement constraints can be imposed by limiting the nodal displacements. For frame structures, the internal forces may vary along the members and element loads (e.g. distributed loads) or bending moments at the end of the member can cause large deflections along the member. Consequently, the internal resistance and the displacements have to be checked at multiple locations along the member. In a standard finite element analysis, a possible strategy is to refine the finite element mesh by dividing the 
members in multiple elements in order to obtain the forces and displacements at a certain location of the members. In the MILP reformulation the introduction of multiple elements not only leads to additional continuous force variables, but also to additional equilibrium equations and member stiffness relation constraints which have to be taken into account. The optimization problem thus becomes large when this approach is adopted.

In this subsection, an approach is proposed to limit the relevant displacement components at predefined locations of the members without the introduction of extra elements by refining the finite element mesh. These displacement constraints are imposed using shape functions that interpolate the nodal displacements in order to calculate the displacements at predefined locations of the members. In the following section, a similar approach is proposed in order to limit the relevant internal stresses at predefined locations of the members.

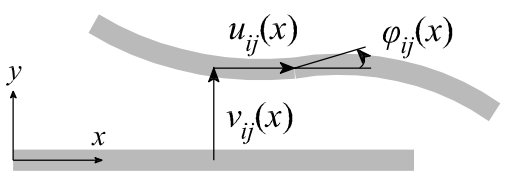

Figure 2: Local displacements

The displacements in the local coordinate system as defined in figure 2 at location $x$ of member $i$ are calculated as:

$$
\begin{aligned}
u_{i j}(x) & =\mathbf{N}_{i}^{u}(x) \mathbf{T}_{i} \mathbf{L}_{i} \mathbf{u}+\tilde{u}_{i j}(x) \\
v_{i j}(x) & =\mathbf{N}_{i}^{v}(x) \mathbf{T}_{i} \mathbf{L}_{i} \mathbf{u}+\tilde{v}_{i j}(x) \\
\varphi_{i j}(x) & =\mathbf{N}_{i}^{\varphi}(x) \mathbf{T}_{i} \mathbf{L}_{i} \mathbf{u}+\tilde{\varphi}_{i j}(x)
\end{aligned}
$$

where the location $x$ is defined along the local $\mathrm{x}$-axis of the member and can have a minimum value of 0 representing the first end of member $i$, and a maximum value of $L_{i}$ representing the second end of member $i . u_{i j}(x), v_{i j}(x)$ and $\varphi_{i j}(x)$ are respectively the displacement along the local $\mathrm{x}$-axis, the displacement along the local $\mathrm{y}$-axis and the rotation at location $x$ of member $i$ for profile $j$ as shown in figure 2 , and $\tilde{u}_{i j}(x), \tilde{v}_{i j}(x)$ and $\tilde{\varphi}_{i j}(x)$ are the displacements at location $x$ of member $i$ for profile $j$ for the case of the beam with clamped-clamped boundary conditions subjected to the element loads. The shape function vectors $\mathbf{N}_{i}^{u}(x), \mathbf{N}_{i}^{v}(x)$ and $\mathbf{N}_{i}^{\varphi}(x)$ are given by:

$$
\begin{aligned}
& \mathbf{N}_{i}^{u}(x)=\left[\begin{array}{llllll}
1-\frac{x}{L_{i}} & 0 & 0 & \frac{x}{L_{i}} & 0 & 0
\end{array}\right] \\
& \mathbf{N}_{i}^{v}(x)=\left[0\left[1-\frac{3 x^{2}}{L_{i}^{2}}+\frac{2 x^{3}}{L_{i}^{3}}\right] \quad\left[x\left(1-\frac{x}{L_{i}}\right)^{2}\right] \quad 0\left[\frac{3 x^{2}}{L_{i}^{2}}-\frac{2 x^{3}}{L_{i}^{3}}\right] \quad\left[x\left(-\frac{x}{L_{i}}+\frac{x^{2}}{L_{i}^{2}}\right)\right]\right]
\end{aligned}
$$

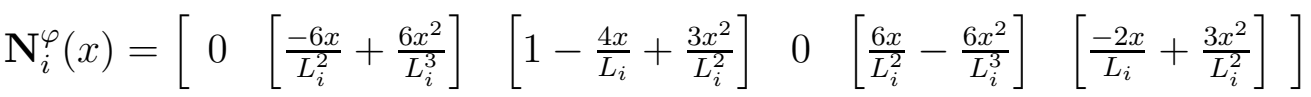

The relevant displacement components $u_{i j}, v_{i j}$ and/or $\varphi_{i j}$ of element $i$ with section $j$ at the output locations $x_{k}$ of interest are collected in a vector $\mathbf{d}_{i j}$. For example, if the transverse displacement $v_{i}$ of element $i$ must be checked at the locations $x_{1}, x_{2}$, and $x_{3}$, the vector $\mathbf{d}_{i j}$ is given by:

$$
\mathbf{d}_{i j}=\left[\begin{array}{c}
v_{i j}\left(x_{1}\right) \\
v_{i j}\left(x_{2}\right) \\
v_{i j}\left(x_{3}\right)
\end{array}\right]
$$


This vector is obtained as follows:

$$
\mathbf{d}_{i j}=\mathbf{N}_{i} \mathbf{T}_{i} \mathbf{L}_{i} \mathbf{u}+\tilde{\mathbf{d}}_{i j}
$$

where $\mathbf{N}_{i}$ is in this case composed as:

$$
\mathbf{N}_{i}=\left[\begin{array}{l}
\mathbf{N}_{i}^{v}\left(x_{1}\right) \\
\mathbf{N}_{i}^{v}\left(x_{2}\right) \\
\mathbf{N}_{i}^{v}\left(x_{3}\right)
\end{array}\right]
$$

and $\tilde{\mathbf{d}}_{i j}$ as:

$$
\tilde{\mathbf{d}}_{i j}=\left[\begin{array}{c}
\tilde{v}_{i j}\left(x_{1}\right) \\
\tilde{v}_{i j}\left(x_{2}\right) \\
\tilde{v}_{i j}\left(x_{3}\right)
\end{array}\right]
$$

In order to limit the relevant displacement components at all predefined locations of member $i$ the following constraints are introduced:

$$
\underline{\mathbf{d}}_{i j}^{\prime}+\left(\underline{\mathbf{d}}_{i j}-\underline{\mathbf{d}}_{i j}^{\prime}\right) y_{i j} \leqslant \mathbf{N}_{i} \mathbf{T}_{i} \mathbf{L}_{i} \mathbf{u}+\tilde{\mathbf{d}}_{i j} \leqslant \overline{\mathbf{d}}_{i j}^{\prime}+\left(\overline{\mathbf{d}}_{i j}-\overline{\mathbf{d}}_{i j}^{\prime}\right) y_{i j} \quad \forall i \in \mathcal{M}, \forall j \in \mathcal{C}_{i}
$$

where $\underline{\mathbf{d}}_{i j}$ and $\overline{\mathbf{d}}_{i j}$ are the prescribed minimum and maximum allowed value of displacements, respectively, and $\underline{\mathbf{d}}_{i j}^{\prime}$ and $\overline{\mathbf{d}}_{i j}^{\prime}$ are artificial lower and upper bounds, respectively, in order to ensure feasibility when profile $j$ is not selected for member $i$. When profile $j$ is selected for member $i\left(y_{i j}=1\right)$ equation (23) becomes $\underline{\mathbf{d}}_{i j} \leqslant \mathbf{N}_{i} \mathbf{T}_{i} \mathbf{L}_{i} \mathbf{u}+\tilde{\mathbf{d}}_{i j} \leqslant \overline{\mathbf{d}}_{i j}$ and the relevant displacement components are limited. When profile $j$ is not selected for member $i\left(y_{i j}=0\right)$ equation (23) reduces to $\underline{\mathbf{d}}_{i j}^{\prime} \leqslant \mathbf{N}_{i} \mathbf{T}_{i} \mathbf{L}_{i} \mathbf{u}+\tilde{\mathbf{d}}_{i j} \leqslant \overline{\mathbf{d}}_{i j}^{\prime}$. The lower bounds $\underline{\mathbf{d}}_{i j}^{\prime}$ and upper bounds $\overline{\mathbf{d}}_{i j}^{\prime}$ are calculated as:

$$
\begin{aligned}
\underline{\mathbf{d}}_{i j}^{\prime}=\min _{\mathbf{u}} & \mathbf{N}_{i} \mathbf{T}_{i} \mathbf{L}_{i} \mathbf{u}+\tilde{\mathbf{d}}_{i j} \\
\text { s.t. } & \underline{\mathbf{u}} \leqslant \mathbf{u} \leqslant \overline{\mathbf{u}} \\
\overline{\mathbf{d}}_{i j}^{\prime}=\max _{\mathbf{u}} & \mathbf{N}_{i} \mathbf{T}_{i} \mathbf{L}_{i} \mathbf{u}+\tilde{\mathbf{d}}_{i j} \\
\text { s.t. } & \underline{\mathbf{u}} \leqslant \mathbf{u} \leqslant \overline{\mathbf{u}}
\end{aligned}
$$

where $\underline{\mathbf{u}}$ and $\overline{\mathbf{u}}$ are the minimum and maximum allowed nodal displacements, respectively.

When only nodal displacements are limited, the constraints given by equation (23) can be substituted by the following constraints:

$$
\underline{\mathbf{u}} \leqslant \mathbf{u} \leqslant \overline{\mathbf{u}}
$$

\subsection{Stresses}

The resistance of cross-sections subjected to shear forces, normal forces, and bending moments must be checked at predefined locations of the members. For elastic design, the resistance constraints can be written in terms of stresses as:

$$
\begin{gathered}
\sigma_{\min } \leqslant \sigma_{\mathrm{t}, i j}(x) \leqslant \sigma_{\max } \\
\sigma_{\min } \leqslant \sigma_{\mathrm{b}, i j}(x) \leqslant \sigma_{\max } \\
\tau_{\min } \leqslant \tau_{i j}(x) \leqslant \tau_{\max }
\end{gathered}
$$




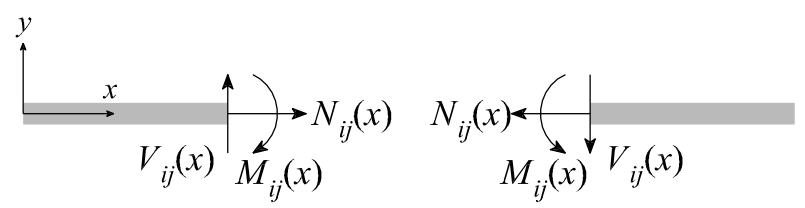

Figure 3: Internal forces of member $i$ for profile $j$ at location $x$

where $\sigma_{\mathrm{t}, i j}(x), \sigma_{\mathrm{b}, i j}(x)$, and $\tau_{i j}(x)$ are the normal stress at the top, the normal stress at the bottom and the maximum shear stress of member $i$ for profile $j$ at location $x$. These stresses are calculated as

$$
\begin{aligned}
\sigma_{\mathrm{t}, i j}(x) & =\frac{N_{i j}(x)}{A_{i j}}+\frac{M_{i j}(x)}{W_{\mathrm{t}, i j}} \\
\sigma_{\mathrm{b}, i j}(x) & =\frac{N_{i j}(x)}{A_{i j}}-\frac{M_{i j}(x)}{W_{\mathrm{b}, i j}} \\
\tau_{i j}(x) & =\frac{V_{i j}(x) S_{i j}}{I_{i j} b_{i j}}
\end{aligned}
$$

where $W_{\mathrm{t}, i j}$ is the section modulus at the top, $W_{\mathrm{b}, i j}$ is the section modulus at the bottom, $S_{i j}$ is the first moment of area, $I_{i j}$ is the second moment of area, and $b_{i j}$ is the width of the profile at the point where the maximum shear stress occurs. $N_{i j}(x), V_{i j}(x)$, and $M_{i j}(x)$ represent, respectively, the normal force, shear force, and bending moment at location $x$ as given by figure 3. For plastic design, similar constraints related to the stress resultants can be formulated.

The internal stresses at location $x$ of member $i$ for profile $j$ are calculated as:

$$
\begin{aligned}
\sigma_{\mathrm{t}, i j}(x) & =\mathbf{B}_{i j}^{\sigma_{\mathrm{t}}}(x) \mathbf{T}_{i} \mathbf{L}_{i} \mathbf{u}+\tilde{\sigma}_{\mathrm{t}, i j}(x) \\
\sigma_{\mathrm{b}, i j}(x) & =\mathbf{B}_{i j}^{\sigma_{\mathrm{b}}}(x) \mathbf{T}_{i} \mathbf{L}_{i} \mathbf{u}+\tilde{\sigma}_{\mathrm{b}, i j}(x) \\
\tau_{i j}(x) & =\mathbf{B}_{i j}^{\tau}(x) \mathbf{T}_{i} \mathbf{L}_{i} \mathbf{u}+\tilde{\tau}_{i j}(x)
\end{aligned}
$$

where $\tilde{\sigma}_{\mathrm{t}, i j}(x), \tilde{\sigma}_{\mathrm{b}, i j}(x)$ and $\tilde{\tau}_{i j}(x)$ are the stresses of the beam with clamped-clamped boundary conditions subjected to the element loads at location $x$ of member $i$ for profile $j$. They are calculated as:

$$
\begin{aligned}
\tilde{\sigma}_{\mathrm{t}, i j}(x) & =\frac{\tilde{N}_{i}(x)}{A_{i j}}+\frac{\tilde{M}_{i}(x)}{W_{\mathrm{t}, i j}} \\
\tilde{\sigma}_{\mathrm{b}, i j}(x) & =\frac{\tilde{N}_{i}(x)}{A_{i j}}-\frac{\tilde{M}_{i}(x)}{W_{\mathrm{b}, i j}} \\
\tilde{\tau}_{i j}(x) & =\frac{\tilde{V}_{i}(x) S_{i j}}{I_{i j} b_{i j}}
\end{aligned}
$$

where $\tilde{N}_{i}(x), \tilde{V}_{i}(x)$ and $\tilde{M}_{i}(x)$ are respectively the normal forces, shear forces and bending moments of the beam with clamped-clamped boundary conditions subjected to the element loads at location $x$ of member $i$. Starting from the given displacements $\mathbf{u}$ the stresses can be 
calculated using the vectors $\mathbf{B}_{i}^{\sigma_{\mathrm{t}}}(x), \mathbf{B}_{i}^{\sigma_{\mathrm{b}}}(x)$ and $\mathbf{B}_{i}^{\tau}(x)$ :

$$
\begin{aligned}
& \mathbf{B}_{i j}^{\sigma_{\mathrm{t}}}(x)=\left[\begin{array}{llllll}
-\frac{E}{L_{i}} & \frac{6 E I_{i j}}{L_{i}^{2} W_{\mathrm{t}, i j}}-\frac{12 x E I_{i j}}{L_{i}^{3} W_{\mathrm{t}, i j}} & \frac{4 E I_{i j}}{L_{i} W_{\mathrm{t}, i j}}-\frac{6 x E I_{i j}}{L_{i}^{2} W_{\mathrm{t}, i j}} & \frac{E}{L_{i}} & -\frac{6 E I_{i j}}{L_{i}^{2} W_{\mathrm{t}, i j}}+\frac{12 x E I_{i j}}{L_{i}^{3} W_{\mathrm{t}, i j}} & \frac{2 E I_{i j}}{L_{i} W_{\mathrm{t}, i j}}-\frac{6 x E I_{i j}}{L_{i}^{2} W_{\mathrm{t}, i j}}
\end{array}\right] \\
& \mathbf{B}_{i j}^{\sigma_{\mathrm{b}}}(x)=\left[\begin{array}{lllll}
-\frac{E}{L_{i}} & -\frac{6 E I_{i j}}{L_{i}^{2} W_{\mathrm{b}, i j}}+\frac{12 x E I_{i j}}{L_{i}^{3} W_{\mathrm{b}, i j}} \quad-\frac{4 E I_{i j}}{L_{i} W_{\mathrm{b}, i j}}+\frac{6 x E I_{i j}}{L_{i}^{2} W_{\mathrm{b}, i j}} \quad \frac{E}{L_{i}} \quad \frac{6 E I_{i j}}{L_{i}^{2} W_{\mathrm{b}, i j}}-\frac{12 x E I_{i j}}{L_{i}^{3} W_{\mathrm{b}, i j}} \quad-\frac{2 E I_{i j}}{L_{i} W_{\mathrm{b}, i j}}+\frac{6 x E I_{i j}}{L_{i}^{2} W_{\mathrm{b}, i j}}
\end{array}\right] \\
& \mathbf{B}_{i j}^{\tau}(x)=\left[\begin{array}{llllll}
0 & -\frac{12 E S_{i j}}{L_{i}^{3} b_{i j}} & -\frac{6 E S_{i j}}{L_{i}^{2} b_{i j}} & 0 & \frac{12 E S_{i j}}{L_{i}^{3} b_{i j}} & -\frac{6 E S_{i j}}{L_{i}^{2} b_{i j}}
\end{array}\right]
\end{aligned}
$$

The relevant stress components $\sigma_{\mathrm{t}, i j}, \sigma_{\mathrm{b}, i j}$ and/or $\tau_{i j}$ of element $i$ with section $j$ at the selected output locations $x_{k}$ are collected in a vector $\mathbf{s}_{i j}$. For example, if the normal stress at the top of element $i$ must be checked at the locations $x_{1}, x_{2}$, and $x_{3}$, the vector $\mathrm{s}_{i j}$ is given by:

$$
\mathbf{s}_{i j}=\left[\begin{array}{l}
\sigma_{\mathrm{t}, i j}\left(x_{1}\right) \\
\sigma_{\mathrm{t}, i j}\left(x_{2}\right) \\
\sigma_{\mathrm{t}, i j}\left(x_{3}\right)
\end{array}\right]
$$

This vector is obtained as follows:

$$
\mathbf{s}_{i j}=\mathbf{B}_{i j} \mathbf{T}_{i} \mathbf{L}_{i} \mathbf{u}+\tilde{\mathbf{s}}_{i j}
$$

where $\mathbf{B}_{i j}$ is in this case composed as:

$$
\mathbf{B}_{i j}=\left[\begin{array}{c}
\mathbf{B}_{i j}^{\sigma_{\mathrm{t}}}\left(x_{1}\right) \\
\mathbf{B}_{i j}^{\sigma_{\mathrm{t}}}\left(x_{2}\right) \\
\mathbf{B}_{i j}^{\sigma_{\mathrm{t}}}\left(x_{3}\right)
\end{array}\right]
$$

and $\tilde{\mathbf{s}}_{i j}$ as:

$$
\tilde{\mathbf{s}}_{i j}=\left[\begin{array}{c}
\tilde{\sigma}_{\mathrm{t}, i j}\left(x_{1}\right) \\
\tilde{\sigma}_{\mathrm{t}, i j}\left(x_{2}\right) \\
\tilde{\sigma}_{\mathrm{t}, i j}\left(x_{3}\right)
\end{array}\right]
$$

The internal resistance constraints read as:

$$
\underline{\mathbf{s}}_{i j}^{\prime}+\left(\underline{\mathbf{s}}_{i j}-\underline{\mathbf{s}}_{i j}^{\prime}\right) y_{i j} \leqslant \mathbf{B}_{i j} \mathbf{T}_{i} \mathbf{L}_{i} \mathbf{u}+\tilde{\mathbf{s}}_{i j} \leqslant \overline{\mathbf{s}}_{i j}^{\prime}+\left(\overline{\mathbf{s}}_{i j}-\overline{\mathbf{s}}_{i j}^{\prime}\right) y_{i j} \quad \forall i \in \mathcal{M}, \forall j \in \mathcal{C}_{i}
$$

where $\tilde{\mathbf{s}}_{i j}$ is a vector containing the selected stress components at the predefined locations of the beam with clamped-clamped boundary conditions subjected to the element loads of member $i$ for profile $j, \underline{\mathbf{s}}_{i j}$ and $\overline{\mathbf{s}}_{i j}$ are the prescribed minimum and maximum allowed stresses, and $\underline{\mathbf{s}}_{i j}^{\prime}$ and $\overline{\mathbf{s}}_{i j}^{\prime}$ are artificial lower and upper bounds in order to ensure feasibility when profile $j$ is not selected for member $i$, calculated similarly to equations (24) and (25). 


\section{Three-bay three-story frame example problem}

\subsection{Problem description}

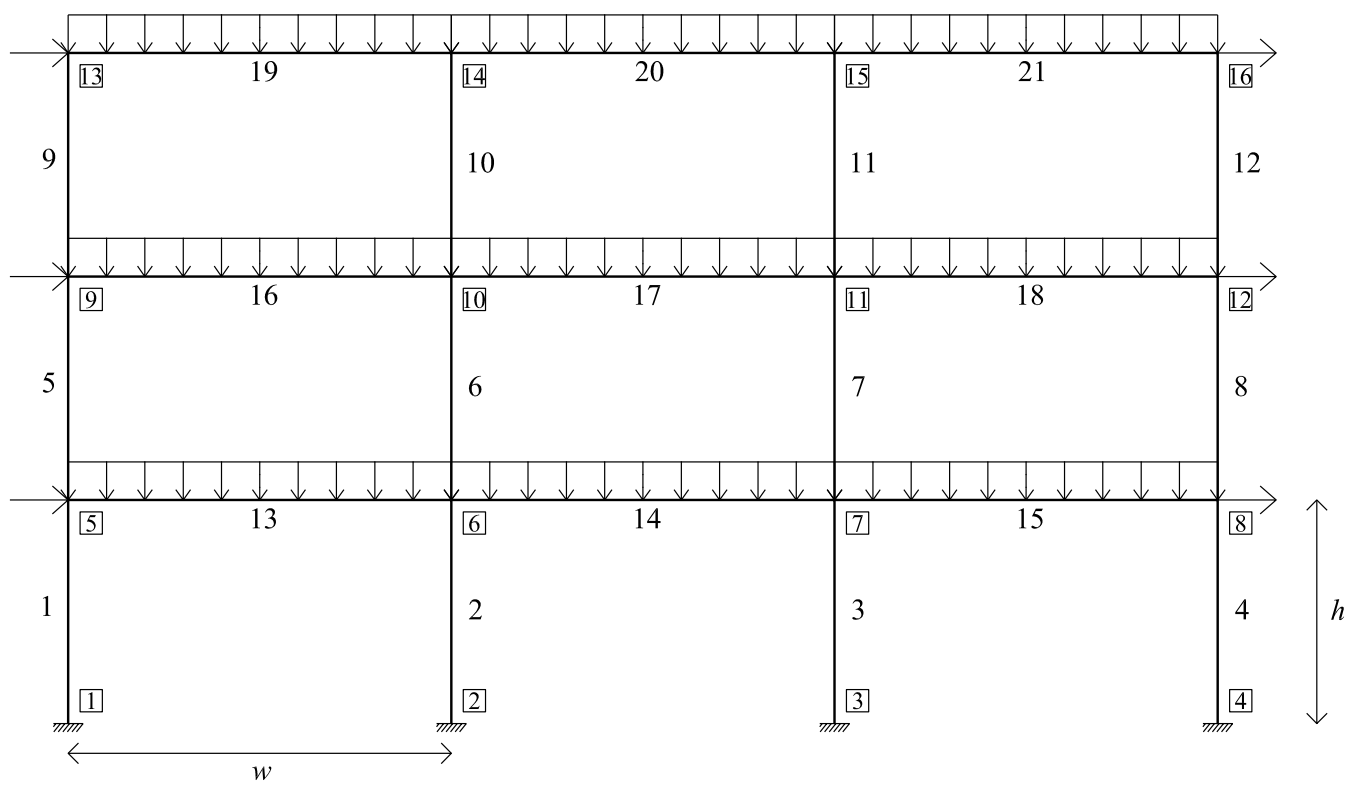

Figure 4: Three-bay three-story frame

Figure 4 shows a three-bay three-story frame structure with twenty-one members. The height of each story is $h=3.5 \mathrm{~m}$, the width of each bay is $w=6 \mathrm{~m}$, the value of the horizontal load is $F=22.05 \mathrm{kN}$, and the value of the distributed vertical load is $p=50.1 \mathrm{kN} / \mathrm{m}$. The members are subdivided in seven groups: the profiles of all beams are the same, and for each story the profiles of the outer columns, and the profiles of the inner columns are the same.

The objective of the optimization problem is to minimize the weight of the structure. In order to reduce the computation time, a limited set of available profiles is used: the profile sections have to be chosen from a HEA catalog, but the range is limited from HEA 100 to HEA 400, meaning 15 alternatives. The Young's modulus of the material is $210 \mathrm{GPa}$, the material density is $7850 \mathrm{~kg} / \mathrm{m}^{3}$, and the yield strength of the material is $f_{\mathrm{y}}=235 \mathrm{MPa}$. The maximum allowed normal stress is $f_{\mathrm{y}}=235 \mathrm{MPa}$, and the maximum allowed shear stress is $f_{\mathrm{y}} / \sqrt{3}=136 \mathrm{MPa}$. For each member, all stress components are limited at three equidistant locations $x_{1}=0, x_{2}=L_{i} / 2$, and $x_{3}=L_{i}$. For each column, the interstory drift $\Delta u$ is limited by $h / 300=0.0117 \mathrm{~m}$. For each beam, the vertical deflection is limited at location $x_{1}=L_{i} / 2$ by $w / 200=0.03 \mathrm{~m}$.

The minimum weight problem is given by equations (3) to (9). The total number of members is $n_{\mathrm{m}}=21$, and for each member the number of available profiles is $n_{\mathrm{s}}=15$, resulting in $n_{\mathrm{b}}=315$ binary decision variables. The number of force variables is $6 n_{\mathrm{b}}=1890$, and the number of degrees of freedom is $n_{\mathrm{dof}}=36$.

\subsection{Results}

The MILP consists of 2241 design variables, 13791 constraints, and 61105 nonzeros in the constraint coefficient matrix. The number of design combinations is $15^{7}=171 \times 10^{6}$. There are 21 equality constraints to ensure that only one profile is selected for each member (given by equation (4)), 36 nodal equilibrium equality constraints (5), 3780 member stiffness relation inequality constraints (6), 3780 force inequality constraints (7), 270 deflection constraints (8), 
5670 stress inequality constraints (9), and 210 grouping constraints. The interstory drift constraints are imposed by limiting the difference of the horizontal nodal displacements at the top and bottom of each column. Consequently, there are 24 interstory drift constraints. The MILP is solved by the software Gurobi 6.0.2 [23] on a computer with an Intel Core i7-5600U processor (2.6 GHz clock frequency) and $8 \mathrm{~GB}$ RAM. The feasibility tolerance is set to $10^{-9}$, the integer feasibility tolerance is set to $10^{-9}$, and the optimality gap is set to $5 \times 10^{-3}$. The optimization problem is solved in 74781 seconds or 21 hours, and 1764305 nodes of the branch-and-bound tree are explored. The optimum design is given in figure 5. The total weight of the structure is $6131.87 \mathrm{~kg}$.

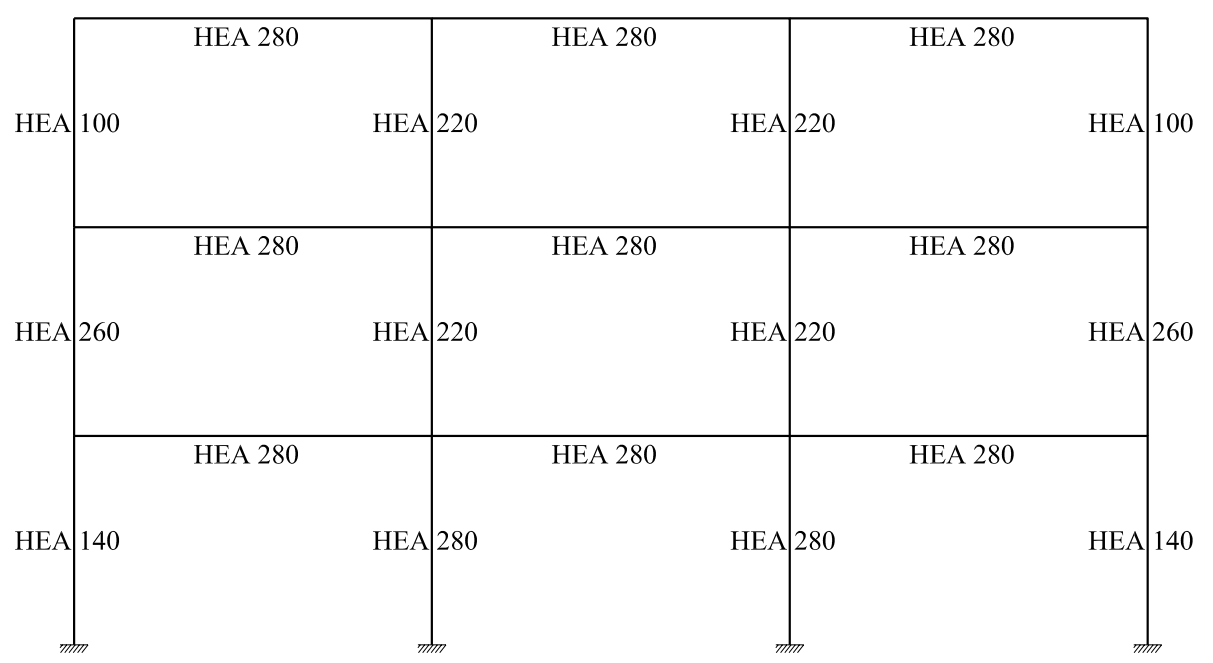

Figure 5: Optimal design

\section{Conclusion}

A method is proposed for the discrete size optimization of frames to global optimality. The optimization problem is reformulated as a mixed-integer linear program and solved with the branch-and-bound method in order to guarantee global optimality. The design variables consist of binary decision variables, continuous displacements, and continuous member end force variables. The equilibrium of the structure is imposed by nodal equilibrium constraints. The relation between the forces and the displacements is given by the member stiffness relation constraints. The relevant displacement components as well as the internal stresses are limited at several predefined locations by interpolating the nodal displacements using shape functions.

In this paper, only member resistance constraints and displacement constraints are considered. It is hard to consider all relevant code constraints, since not all constraints can be reformulated as linear constraints. Member stability constraints are therefore not taken into account. More research is needed to develop a fast optimization method that can take all relevant code constraints into account and that can solve discrete optimization problems to global optimality. 


\section{REFERENCES}

[1] J. Farkas. Structural optimization as a harmony of design, fabrication and economy. Structural and Multidisciplinary Optimization, 30:66-75, 2005.

[2] Q. Wang and J. S. Arora. Alternative formulations for structural optimization: An evaluation using frames. Journal of Structural Engineering, 132(12):1880-1189, 2006.

[3] P.B. Thanedar and G.N. Vanderplaats. Survey of discrete variable optimization for structural design. Journal of Structural Engineering, 121:301-306, 1995.

[4] J.S. Arora and M.-W. Huang. Discrete structural optimization with commercially available sections. Structural Engineering/Earthquake Engineering, 13:93-110, 1996.

[5] M.-W. Huang and J.S. Arora. Optimal design of steel structures using standard sections. Structural Optimization, 14:24-35, 1997.

[6] J. Arora. Methods for discrete variable structural optimization. In Scott A. Burns, editor, Recent Advances in Optimal Structural Design, pages 1-40. ASCE, 2002.

[7] D.S. Juang and W.T. Chang. A revised discrete lagrangian-based search algorithm for the optimal design of skeletal structures using available sections. Structural and Multidisciplinary Optimization, 31:201-210, 2006.

[8] M. Schevenels, S. McGinn, A. Rolvink, and J. Coenders. An optimality criteria based method for discrete design optimization taking into account buildability constraints. Structural and Multidisciplinary Optimization, 50(5):755-774, 2014.

[9] R.J. Balling. Optimal steel frame design by simulated annealing. Journal of structural engineering, 117(6):1780-1795, 1991.

[10] C. Camp, S. Pezeshk, and C. Guozhong. Optimized design of two-dimensional structures using a genetic algorithm. Journal of structural engineering, 124(5):551-559, 1998.

[11] S. Rajeev and C.S. Krishnamoorthy. Discrete optimization of structures using genetic algorithms. Journal of Structural Engineering, 118(5):1233-1250, 1992.

[12] C.V. Camp, B.J. Bichon, and S.P. Stovall. Design of steel frames using ant colony optimization. Journal of structural engineering, 131(7):369-379, 2005.

[13] A.H. Gandomi, X.S. Yang, and A.H. Alavi. Mixed variable structural optimization using firefly algorithm. Computers \& Structures, 89(23):2325-2336, 2011.

[14] M. Sonmez. Discrete optimum design of truss structures using artificial bee colony algorithm. Structural and Multidisciplinary Optimization, 43(1):85-97, 2011.

[15] G. Venter and J. Sobieszczanski-Sobieski. Particle swarm optimization. AIAA Journal, 41(8):1583-1589, 2003.

[16] J.S. Arora and Q. Wang. Review of formulations for structural and mechanical system optimization. Structural and Multidisciplinary Optimization, 30(4):251-272, 2005. 
[17] I.E. Grossmann, V.T. Voudouris, and O. Ghattas. Mixed-integer linear programming reformulations for some nonlinear discrete design optimization problems. In C.A. Floudas and P.M. Pardalos, editors, Recent advances in global optimization, pages 478-512. Princeton University Press, 1992.

[18] M.H. Rasmussen and M. Stolpe. Global optimization of discrete truss topology design problems using a parallel cut-and-branch method. Computers \& Structures, 86(13):15271538, 2008.

[19] K. Mela. Resolving issues with member buckling in truss topology optimization using a mixed variable approach. Structural and Multidisciplinary Optimization, 50(6):10371049, 2014.

[20] R. Van Mellaert, G. Lombaert, and M. Schevenels. Global size optimization of statically determinate trusses considering displacement, member, and joint constraints. Journal of Structural Engineering, 2015. Published online.

[21] A. Kassimali. Matrix Analysis of Structures. Brooks/Cole Publishing Company, London, UK, 1999.

[22] M. Stolpe and K. Svanberg. Modelling topology optimization problems as linear mixed 0-1 programs. International Journal for Numerical Methods in Engineering, 57:723-739, 2003.

[23] Inc. Gurobi Optimization. Gurobi optimizer reference manual, 2015. 